\title{
Thrombin-Antithrombin III-Komplex (TAT): Bestimmung nun auch im Notfall möglich
}

\section{Thrombin-Antithrombin III-Complex: Determination now Possible in Emergency Cases}

\author{
H. D. Bruhn', J. Liebsch', C. Wagner ${ }^{2}$ \\ 1 Medizinische Klinik, Universität Kiel, ${ }^{2}$ Behringwerke AG, Marburg
}

\begin{abstract}
Zusammenfassung:
Die vorliegenden Daten zeigen, daß mit einer modifizierten, zeitverkürzten Version eines Enzymimmunoassays die Bestimmung von Thrombin-Antithrombin III-Komplex (TAT) innerhalb von 20 min durchgeführt werden kann. Die oft therapieentscheidende Diagnose einer Hyperkoagulabilität kann somit auch im Notfall gestellt werden.
\end{abstract}

Schlüsselwörter:

Thrombin-Antithrombin III (TAT) - Hyperkoagulabilität - Notfallmethode

Summary:

The presented data show, that thrombin-antithrombin III-complex (TAT) determination is now possible within $20 \mathrm{~min}$ by a modified time reduced version of an enzymimmunoassay. The diagnosis of hypercoagulability, often decisive for the following therapy, now can be also made in emergency cases.

Keywords:

Thrombin-Antithrombin III (TAT) - hypercoagulability - emergency method

\section{Einleitung}

Die Bestimmung des Thrombin-Antithrombin III-Komplex (TAT) stellt ein quantitatives Maß der In-vivo-Gerinnungsaktivierung dar (1). Die Aktivierung des Prothrombin zu Thrombin ist die Schlüsselreaktion in der Gerinnungskaskade; die Inaktivierung des Thrombin mittels Komplexierung mit AT III, dem wichtigsten physiologischen Gerinnungsinhibitor, stellt somit ein indirektes Maß der Thrombinbildung im Blutstrom dar.

Gerinnungsstörungen durch Verbrauchskoagulopathie stellen in der Akutmedizin eine der gefürchtetsten, weil den Patienten letal bedrohenden Komplikationen dar, wobei sowohl Blutungen als auch ein Multiorganversagen infolge der Mikrothrombosierung des Kapillarnetzes Todesursache sein können. In beiden Fällen jedoch resultieren die Komplikationen aus einer gesteigerten Thrombinaktivität, die im Mittelpunkt der als disseminierte intravasale Koagulopathie (DIC) bezeichneten Gerinnungsstörung mit einem erhöhten Umsatz an Thrombozyten, Fibrinogen und Gerinnungsfaktoren sowie Inhibitoren steht.

Mit dem Nachweis einer gesteigerten Thrombinaktivie-. rung wird die Diagnose der DIC wesentlich erleichtert (2, 3), da die Analyse der üblichen Gerinnungsparameter ein völlig unterschiedliches Bild je nach Ausprägungsart der DIC ergeben können. Erstrebenswert ist insbesondere eine möglichst frühzeitige Erfassung der auftretenden Hyperkoagulabilität, um durch eine rechtzeitig einsetzende Therapie die volle Ausildung einer DJC zu verhindern.

Bei der TAT-Bestimmung handelt es sich um einen Enzymimmunoassay im Sandwichprinzip; die übliche Testdauer beträgt ca. $2 \mathrm{~h}$. Um die gerade in der Akutmedizin wertvolle Information über den In-vivo-Aktivitätsgrad des Gerinnungssystems schneller zu erhalten, wurde eine auf 20 min Inkubationszeit insgesamt verkürzte Testversion auf ihre Vergleichbarkeit mit der Standardversion geprüft.

\section{Material und Methoden}

TAT wurde bestimmt mittels Enzygnost ${ }^{\circledR}$ TAT (Behringwerke AG, 3550 Marburg). Die Testdurchführung erfolgt entweder gemäß Packungsbeilage (Standardversion) oder gemäß der folgenden Schnellversion:

Je beschichtetes Röhrchen:

$100 \mu$ l Probenpuffer

$200 \mu$ l Probe (oder Standard)

$10 \mathrm{~min}$ bei $37^{\circ} \mathrm{C}$ inkubièren

$2 \times$ waschen

$300 \mu$ l Konjugat-Lösung (1:20 verd.)

$5 \mathrm{~min}$ bei $37^{\circ} \mathrm{C}$ inkubieren

$3 \times$ waschen

$300 \mu$ l Substrat-Lösung

$5 \mathrm{~min}$ bei $37^{\circ} \mathrm{C}$ inkubieren

$300 \mu$ l Stopplösung $\cdots$

Für die Schnellversion wurden Einzelbestimmungen, für die Standardversion Doppelbestimmungen durchgeführt. In jeder Meßserie wurde eine Standardreihe zur Auswertung mitgeführt. Die Auswertung erfolgte mittels polygonaler-Interpolation: Als Probenmaterial wurden ausschließlich Citratplasmaproben verwendet (1 Teil Citratlösung +9 Teile Venenblut).

\section{Ergebnisse}

\section{Präzision}

Die im. Test-Kit enthaltene Kontrolle sowie 3 unterschiedliche Plasmapools wurden in einer Serie mehrfach be- 


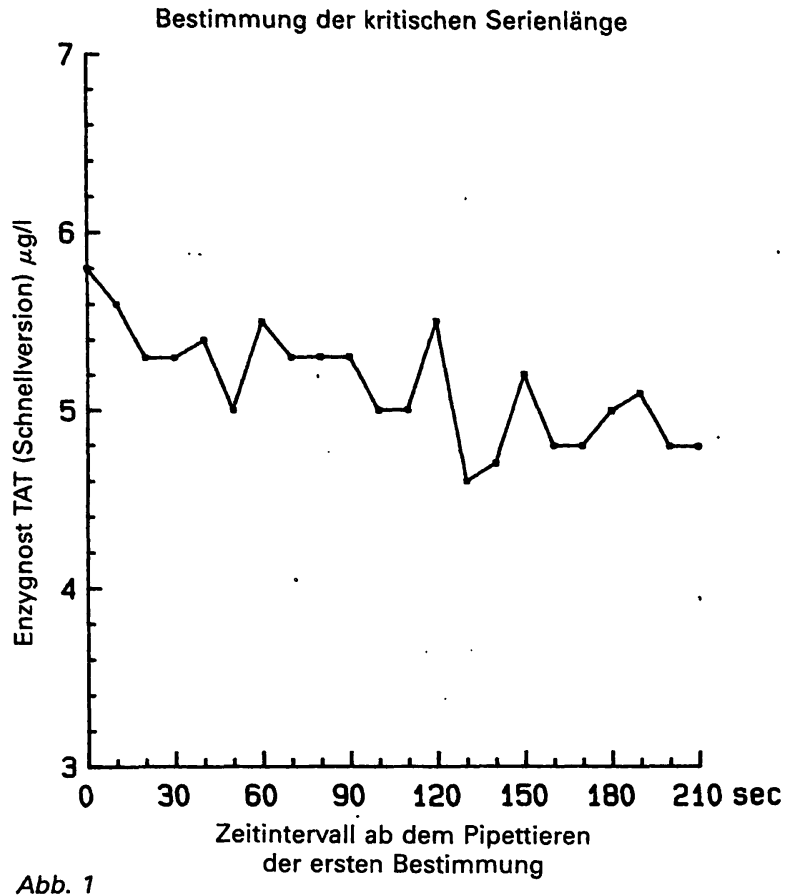

Abb. 1

Tab. 1: Präzision

\begin{tabular}{|c|c|c|c|c|c|c|}
\hline & \multicolumn{3}{|c|}{ intra-assay } & \multicolumn{3}{|c|}{ inter-assay } \\
\hline & $\underset{(\mu g / l)}{\overline{\mathbf{x}}}$ & $\underset{(\mu \mathrm{g} / l)}{\mathbf{S}}$ & $\begin{array}{l}\text { VK } \\
(\%)\end{array}$ & $\underset{(\mu g / l)}{\bar{x}}$ & $\underset{(\mu g / l)}{\mathbf{S}}$ & $\begin{array}{l}\text { VK } \\
(\%)\end{array}$ \\
\hline $\begin{array}{l}\text { Kontrolle }(n=18) \\
\text { Plasmapool I }(n=10) \\
\text { Plasmapool II }(n=10) \\
\text { Plasmapool III }(n=10)\end{array}$ & $\begin{array}{r}10,2 \\
5,7 \\
9,7 \\
20,3\end{array}$ & $\begin{array}{l}1,3 \\
0,9 \\
1,4 \\
2,5\end{array}$ & $\begin{array}{l}12,6 \\
16,0 \\
15,5 \\
12,1\end{array}$ & $\begin{array}{r}10,3 \\
5,1 \\
8,1 \\
25,6\end{array}$ & $\begin{array}{l}0,9 \\
1,0 \\
0,8 \\
4,4\end{array}$ & $\begin{array}{r}8,4 \\
20,4 \\
9,5 \\
17,0\end{array}$ \\
\hline
\end{tabular}

$\overline{\mathrm{x}}=$ Mittelwert

$s=$ Standardabweichung

VK $=$ Variationskoeffizient

stimmt (Intra-assay-Präzision) sowie in 10 verschiedenen Serien (Inter-assay Präzision). Tabelle 1 zeigt die erhaltenen Präzisionsdaten. Mit einem mittleren Variationskoeffizienten (VK) von ca. 12=15\% zeigt die auf 20 min verkürzte Testversion eine akzeptable Reproduzierbarkeit.

\section{Kritische Serienlänge}

Aufgrund der kurzen Inkubationszeit und damit einer nicht abgeschlossenen Bindungsreaktion ist für längere Sicht in der Schnellversion eine systematische Verschiebung der Ergebnisse vorstellbar: Um die kritische Serienlänge zu bestimmen, wurde ein Probenplasma in Abständen von $10 \mathrm{sec}$ mehrfach in einer Serie eingesetzt.

Die Auftragung der Ergebnisse über die Zeit (Abb. 1) zeigt eine gute Konstanz der Ergebnisse bis zu einer $\mathrm{Pi}$ pettierdauer von $120 \mathrm{sec}$; bei einem errechneten Mittelwert von $5,33 \mu \mathrm{g} / \mathrm{I}$ TAT wurde ein VK von $4,5 \%$ erhalten. Später pipettierte Proben zeigten erniedrigte Resultate $(\bar{x}$ für $130-210 \mathrm{sec}: 4,86 \mu \mathrm{g} / \mathrm{l}$ TAT). Die gesamte Pipettierdauer der Standards und Proben sollte daher 2 min nicht überschreiten. Diese Zeit ist für das Pipettieren von 6-10 Proben gut praktikabel.

\section{Methodenvergleich}

100 Patientenproben aus der täglichen Routine wurden parallel in der Standard- und Schnellversion getestet 143
TAT-Bestimmung

Methodenvergleich

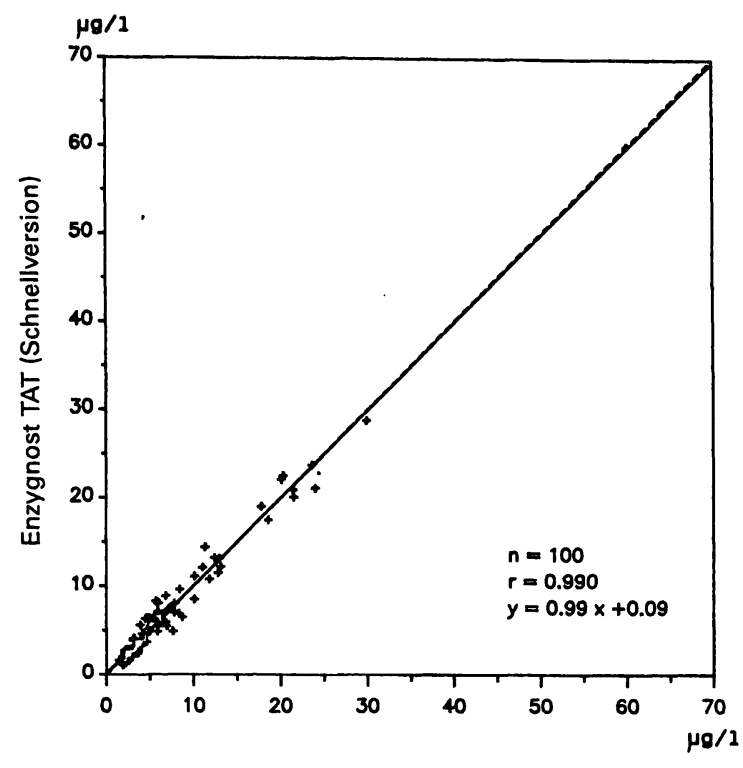

Abb. 2

Enzygnost TAT (Standardversion)

Proben TAT < 4,1 $\mu \mathrm{g} / \mathrm{l} ; 40$ Proben TAT 4,1-10,0 $\mu \mathrm{g} / \mathrm{l} ; 17$ Proben $>10,0 \mu \mathrm{g} / \mathrm{l})$. Der in Abb. 2 gezeigte Vergleich der mit der Standardversion und der Schnellversion erhaltenen TAT-Werte zeigt eine gute Vergleichbarkeit der beiden Methoden; der Regressionskoeffizient beträgt 0,990, die Regressionsgerade weicht nicht signifikant von der $x=y-$ Linie ab.

\section{Zusammenfassung}

Wie die vorgelegten Daten zeigen, ist die Durchführung der TAT-Bestimmung mittels einer zeitverkürzten Testversion des Standard-ELISA innerhalb von $20 \mathrm{~min}$ bei zufriedenstellender Präzision durchführbar. Die mittels Standard- und Schnellversion erhaltenen Ergebnisse zeigten keine statistisch signifikanten Unterschiede. Damit ergibt sich die Möglichkeit der Erfassung des Thrombineinbruchs in die Blutbahn mit Hilfe einer Schnell-Methode, so daß die therapeutisch entscheidende Absicherung der Diagnose Verbraúchskoagulopathie nun auch unter den Bedingungen des Notfall-Labors gewährleistet ist.

Schrifttum:

1. PELZER, H., SCHWARZ, A., HEIMBURGER, N.: Determination of human thrombinantithrombin III complex in plasma with an enzymelinked immunosorbent assay. Thromb Haemostasis 59, 101- 106 (1988).

2. SEITZ, R., EGBRING, R., WAGNER, C., DATI, F.: Thrombin-Antithrombin III-Komplex (TAT): Ein Marker für intravasale Gerinnungsaktivierung. Internist 31, 69-74 (1990). 3. HOEK, J. A., STURK, A., TEN CATE, J. W., LAMPING, R. J., BERENDS, F., BORM, J. J.: Laboratory and clinical evaluation of an assay of thrombin-antithrombin III complex. Clin. Chem. 34, 2058-2062 (1988).

$$
11
$$

Anschrift der Verfasser:

Dr. Carola Wagner

Behringwerke AG, Klinische Prüfung Diagnostica

Postfach 1140, 3550 Marburg

Prof. Dr. Hans-Dietrich Bruhn

Jürgen Liebsch

1. Medizinische Klinik

Schittenhelm-Straße 12, 2300 Kiel 